\title{
Maximize the Coverage Lifetime of Sensor Networks ${ }^{\star}$
}

\author{
Minh-Long Pham, Daeyoung Kim, Taehong Kim, and Seong-eun Yoo \\ Real-time and Embedded Systems Laboratory \\ Information and Communications University, Korea \\ \{longpm, kimd, damiano, seyoo\}@icu.ac.kr \\ http://resl.icu.ac.kr
}

\begin{abstract}
When deploying sensors in the field in order to collect useful information, one of the most important issues is how to prolong the lifetime of the network because of energy constraint of the sensors while guaranteeing that every point in the network is covered. In this paper, we propose the formulation of integer linear programming (ILP) model to find the optimal network flow in the sensor fields in order to maximize the network lifetime while maintaining the coverage and connectivity. By dividing the network into grid structure, the problem can become manageable in size and complexity thus can be applied to large network with high number of nodes. The experimental results show that our proposed scheme outperforms previous protocols in terms of coverage lifetime.
\end{abstract}

\section{Introduction}

The lifetime of sensor networks mainly depends on battery energy because sensor nodes usually use a small battery and when they are deployed in the field, it is difficult to replace the battery. So, optimal usage of energy is an important issue in order to prolong the lifetime of a sensor network. Also in sensor networks, where sensor nodes are deployed to monitor an area, there is the need to guarantee that every point in the area is covered by at least one sensor node. The following definitions are required in order to understand the coverage problem and our proposed scheme as well.

Definition 1. The network is said to be connected if any of the active nodes can find the path to transmit the data back to the base station.

Definition 2. The network is said to have area coverage if every point in its area is covered of by at least one active sensor node.

Definition 3. The coverage lifetime is the time for which the network maintains area coverage.

Definition 4. The connectivity lifetime is the time for which the network maintains connectivity.

\footnotetext{
* This research has been partially supported by ITRC project of Korea MIC and BK21 project of Korea MOE.
} 
The coverage lifetime can be considered as the time interval upto the point when the network can not cover some area because of the death of the cell, and the connectivity lifetime as the time interval upto the first loss of connectivity among the nodes to the base station. By taking into account both coverage and connectivity metrics, network lifetime is specified as the time interval during which the network still maintains area coverage and connectivity to make the base node monitor the whole area. As shown later in our model, since the loss of area coverage occurs always before the loss of connectivity. So, we can treat the network lifetime as the coverage lifetime.

In this paper, we propose the network model that enables us to monitor the whole sensor field as well as to maximize the coverage lifetime of the network. The proposed network topology is a grid structure. The network is composed of grid cells and each cell has at least one sensor node. Because each cell can be covered by one sensor node, one node in each cell can sense the environment information of its cell and communicate with other nodes in adjacent cells. Therefore, only one node in the cell is active at once time while other nodes in the cell stay in sleep mode. The reasons we propose such network model are 1) energy conservation and 2) simplification of optimal network flow. First, because one active node in each cell can provide both area coverage and connectivity to the network, other nodes do not need to participate in monitoring the sensor field. Instead, they save the energy staying in sleep mode. When the active node runs out of energy, one of the nodes in sleep mode becomes active node for the purpose of maintaining the area coverage and connectivity. Second, grid structure can simplify the optimization problem and network flow by considering the amount of data between cells instead of between nodes. Since optimization problem focuses on the data flow between cell, the problem can be manageable even if the network size or the number of nodes increases.

Based on the proposed network model, we concentrate on finding the optimal network flow among the sensor nodes that guarantee both area coverage and connectivity. We can solve the optimization problem by analyzing the features of area coverage and connectivity. Integer linear programming is applied to find the network flow among the cells toward the base station to maximize the lifetime coverage of the whole network. The constraints for the problem of integer linear programming is derived by analyzing the features of network such as amount of data and energy consumption.

The remainder of this paper is organized as follows. We describe the related work in section 2. Then, we discuss the coverage problem and the network model in section 3 . Section 4 represents the formulation of the integer linear problem. In section 5, we propose some application scenarios for using the proposed scheme. The experimental results in section 6 show the advantages of the proposed scheme, and section 7 concludes the paper.

\section{Related Work}

\subsection{Integer Linear Programming}

Integer linear programming is a kind of operating researches. This method is used to calculate the optimal result, from the constraints, by the using inequality equation. For example, integer linear programming is used to get the minimal route path under the 
given network topology. The integer linear programming problem can be solved in polynomial time. And we can use the LP_SOLVE program in order to get the result of the integer linear programming formulation.

\subsection{Coverage Problem}

The coverage problem can be divided into area coverage and point coverage. In area coverage, the main purpose is to ensure that every point in the area is covered by at least one sensor. Researches in [1] and [2] proposed algorithms to find the maximum number of disjoint sets, such that each set of sensors can monitor the whole area. The paper [3] represents the mechanism to select an area dominating the minimum set of nodes, such that the selected set covers the area. The idea is to construct a connected dominating set that ensures energy efficient area coverage.

In point coverage, the whole area is divided into discrete set of points, with the purpose of guaranteeing that this set of points is covered. In [4], the network is divided into discrete grid points, and the linear programming problem is formulated to find the deployment of sensors such that every grid point is covered by at least $m$ sensors with minimal cost of sensors. In this paper, we focus on guaranteeing the area coverage in order to monitor the whole sensor field during the coverage lifetime of the network.

\subsection{Coverage Lifetime}

Prolonging the lifetime of coverage for a sensor network is investigated under number of works. In the case of the coverage problems [1] and [2], the main purpose of finding the minimal disjoint set is to maximize the coverage lifetime by reducing the number of working nodes. Only one set among all the disjoint sets wakes up and performs sensing task at a certain point in order to reduce the energy consumption. In [4] and [5], the network is divided into discrete grid points, and the integer linear programming problem is formulated by deciding whether to place a sensor node at each grid point so that the number of nodes is minimal while still covering all the discrete grid points. Another distributed and localized protocol, using probing to select and determine the node scheduling for energy efficient coverage, is proposed in [6].

Our proposed scheme is different from the previous coverage problems above in that our scheme obtains the optimal network flow that prolongs the area coverage lifetime as well as maintains connectivity at the same time. Also by dividing the network into cells, the complexity of the linear problem is reduced considerably, and thus it can be applied to large sensor networks with huge number of sensor nodes.

\section{Problem Description}

We assume that the network has the following characteristics in our proposed scheme.

- Sensor network consists of a number of homogeneous sensor nodes with identical transmission range, sensing radius and initial energy.

- All sensor nodes in the network have identical sensing period. This means that all sensor nodes generate the same amount of sensing data. 
- The network is divided into a grid structure as shown in Figure 1. Each cell can be covered by one sensor node, so at one time only one node in the cell is active while all the other nodes in the cell can stay in sleeping mode.
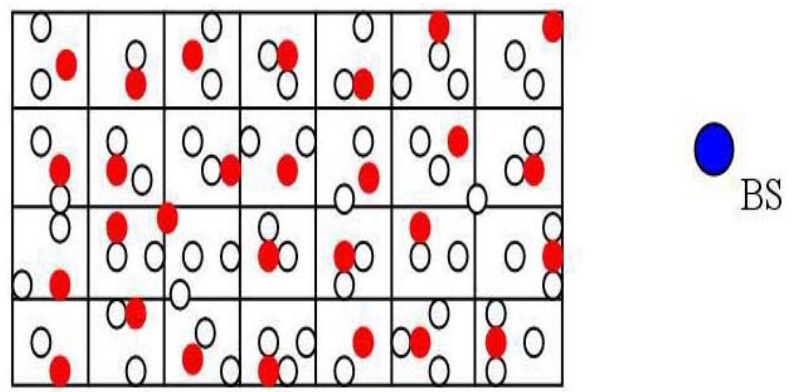

Fig. 1. Grid structure with gray node as the active node in each cell

- To ensure the connectivity, any sensor node in one cell must be connected to any sensor node in one of the adjacent cells as in Figure 2. So, we can derive the relation between transmission range and sensing range as:

$$
\left(R_{T x}\right)^{2}=\left(2 R_{s} / \sqrt{2}\right)^{2}+\left(R_{s} / \sqrt{2}\right)^{2}=5 / 2\left(R_{s}\right)^{2}
$$

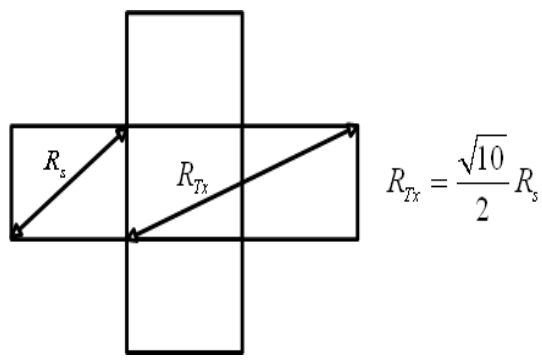

Fig. 2. The relation between transmission and sensing range

- Data gathering period can be divided into rounds, in each round, each active node will generate the same amount of the data for sending to the base station via one or multi hops.

- The network loses area coverage when all the nodes in any one cell are dead.

- One cell is considered at a loss of connectivity when all the adjacent cells are dead (nodes in these adjacent cells are all dead). Thus, cell can not send the data any more.

Since the coverage loss occurs when only one cell is dead whereas the loss of connectivity is occurred when all the adjacent cells are dead, the network will lose coverage 
before losing connectivity. So, the network lifetime is considered as the coverage lifetime until the first cell loses area coverage. Therefore we can define the optimization problem as follows:

Optimization Problem. Given the sensor field (divided into grid structure) and sensor deployment, find the total data flow among the cells to maximize the lifetime until the first cell loses area coverage (all the nodes in that cell are dead).

\section{Problem Formulation}

In this section, we find the several constraints required to formulate the optimal network flow problem. As we assumed in the problem description section, we consider all the sensors have the same initial energy, same radio transmission and sensing range. However, we do not consider the energy consumption for sensing, since it is significantly smaller than the energy consumption for transmission.

The constraints for optimal problem are obtained from consideration of the network characteristics such as data flow between the cells, data flow between the cell and the base station, and energy consumption in each cell.

The notations used in the optimization problem are described in table 1 and we have the following constraints:

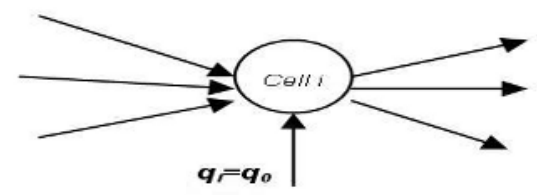

Fig. 3. Data Flow

\section{The conservation of the total data flow at each cell $i$ up to time $T$ is given by}

$$
\begin{gathered}
q_{0} T+\sum_{\substack{m=1 \\
m \neq i}}^{N} V_{m i}=\sum_{\substack{j=1 \\
j \neq i}}^{N+1} V_{i j} \\
(\mathrm{i}=1 \ldots \mathrm{N}, \text { with } \mathrm{N}+1 \text { stands for } \mathrm{BS})
\end{gathered}
$$

The sum of the total data generated by cell $\mathrm{i}$ and the total data coming into cell $\mathrm{i}$ is equal to the total data coming out of cell $\mathrm{i}$ (from cell $\mathrm{i}$ to the adjacent cells and to the BS).

In the above equation, the first monomial $q_{0} T$ is the amount of data sensed by cell $\mathrm{i}$, the second monomial is the amount of data coming into cell $\mathrm{i}$, and the monomial at the right side stands for the amount of data coming out of cell i. Figure 3 shows the data flow of cell i: 
Table 1. Notations

\begin{tabular}{|c|l|}
\hline Term & \multicolumn{1}{|c|}{ Definition } \\
\hline $\mathrm{N}$ & Number of cells \\
\hline$k_{i}$ & Number of nodes in cell $\mathrm{i}$ \\
\hline$R_{T x}$ & Radio transmission range \\
\hline$R_{s}$ & Sensing range \\
\hline$q_{0}$ & $\begin{array}{l}\text { Data rate that each active sensor in a cell collects and generates in one } \\
\text { unit of time }\end{array}$ \\
\hline$E_{0}$ & Network lifetime until all the sensors in the first cell run out of energy \\
\hline$f_{i j}$ & Initial energy of each node \\
\hline$d_{i j}$ & Data rate sent by node $\mathrm{i}$ to node $\mathrm{j}$ \\
\hline$E_{a m p}$ & Transmit amplifier parameter \\
\hline$V_{i j}$ & Total volume of data sent from cell $\mathrm{i}$ to cell $\mathrm{j}$ up to time $\mathrm{T}$ \\
\hline$E_{l e c}$ & Radio dissipates to run transmitter or receiver circuitry \\
\hline$c_{s}$ & Energy consumption cost for sensing one unit of data \\
\hline$c_{i j}$ & Power consumption cost for transmission from $\mathrm{i}$ to $\mathrm{j}$ \\
\hline$E_{T x}(i, j)$ & Power dissipated at node $\mathrm{i}$ when it is transmitting to node $\mathrm{j}$ \\
\hline$E_{R x}(i, j)$ & Power consumption for receiving \\
\hline & \\
\hline
\end{tabular}

\section{The energy constraint for each cell $\mathbf{i}$ with $k_{i}$ nodes is given by}

$$
E_{l e c} * \sum_{\substack{m=1 \\ m \neq i}}^{N} V_{m i}+\sum_{\substack{j=1 \\ j \neq i}}^{N+1} c_{i j} V_{i j} \leq k_{i} * E_{0}
$$

Total energy consumption, for transmission and receiving, of cell $\mathrm{i}$ up to time $\mathrm{T}$ is less than or equal to the total energy of $\mathrm{k}_{\mathrm{i}}$ nodes in cell $\mathrm{i}$.

Since the transmission range of the nodes among cells are the same and the transmission range between a cell to the $\mathrm{BS}$ depends on the distance between the cell and the BS, we have $c_{i j}=c_{0}(\mathrm{i}=1 . . \mathrm{N}, \mathrm{j}=1 . . \mathrm{N})$

In the above equation, the first monomial is the total energy consumption for receiving data in cell $\mathrm{i}$ during the time $\mathrm{T}$, in that it is a form of multiplication of energy consumption for receiving in one unit and the total amount of received data. At this time, we consider there is no additional circuit to receive data, so $E_{l e c}$ is enough energy 
for receiving. In this manner, we can estimate that the second monomial is the total energy consumption for transmission. The difference is that $c_{i j}$ depends on the distance from $\mathrm{i}$ to $\mathrm{j}$ whereas $E_{l e c}$ is a constant. Finally, the monomial at the right side is calculated by multiplying the number of nodes in cell $i$ and the initial energy. The Above equation ignores the energy consumption for sensing, but we can enhance the constraint if we want to take that into account as the following equation:

$$
E_{l e c} * \sum_{\substack{m=1 \\ m \neq i}}^{N} V_{m i}+\sum_{\substack{j=1 \\ j \neq i}}^{N+1} c_{i j} V_{i j}+c_{s} q_{0} T \leq k_{i} * E_{0}
$$

\section{Constraint that all the collected data from the sensor nodes is gathered at BS}

In a sensor network, all the collected data from the sensor field is gathered at the BS. In time $\mathrm{T}$, each cell generates $q_{0} \mathrm{~T}$ amount of data, and this data is transferred to the $\mathrm{BS}$, so the total amount of data sent directly from all the cells to the $\mathrm{BS}$ is equal to $\mathrm{N} q_{0} \mathrm{~T}$ and we have following constraint:

$$
\sum_{i=1}^{N} V_{i N+1}=N q_{0} T
$$

From (1), (2), and (3), we have the following linear programming formulation to maximize $\mathrm{T}$ :

$$
\begin{gathered}
q_{0} T+\sum_{\substack{m=1 \\
m \neq i}}^{N} V_{m i}=\sum_{\substack{j=1 \\
j \neq i}}^{N+1} V_{i j} \\
E_{l e c} * \sum_{\substack{m=1 \\
m \neq i}}^{N} V_{m i}+\sum_{\substack{j=1 \\
j \neq i}}^{N+1} c_{i j} V_{i j} \leq k_{i} * E_{0} \\
\sum_{i=1}^{N} V_{i N+1}=N q_{0} T
\end{gathered}
$$

$T \geq 0, V_{m i} \geq 0, V_{i j} \geq 0(\mathrm{~m}=1 . . \mathrm{N}, \mathrm{i}=1 . . \mathrm{N}, \mathrm{j}=1 . . \mathrm{N}+1)$ where $\mathrm{T}, V_{m i}, V_{i j}, \mathrm{~m}=1 . . \mathrm{N}$, $\mathrm{i}=1 . . \mathrm{N}, \mathrm{j}=1 . . \mathrm{N}=1$ are the decision variables and $\mathrm{N}, k_{i}, q_{0}, E_{0}, E_{l e c}, c_{i N+1}, c_{s}, c_{0}$ are the constants.

Since we consider only the transmission between the cells, so the number of constraints and decision variables are proportional to the number of cells, not to the number of nodes; it means that the number of decision variables is not changed as the number of nodes increases. (The number of variables can be calculated as $1+5 N-4 \sqrt{N}$, and the number of constraints is $2 \mathrm{~N}+1$ ). The constraints can be strictly applied that $\mathrm{T}, V_{m i}$, $V_{i j}$ are integers, and then, we have the integer linear programming problem. 


\section{Applications}

After deploying the sensor nodes in the field, they will self-organize, gather the information required to formulate the problem (such as location of all the sensor nodes and the number of nodes in the cell) and send back to the base station(BS). The BS will formulate and solve the optimization problem based on the received information to get the optimal network flow. The schedule of the network flow is then sent back to the sensor nodes in each cell. Each cell will store the amount of data that is needed to be sent to each adjacent cell or the amount of data to send to the BS; which are considered as links for each cell with the capacity of a link being the total data to be sent on this link. At a time, one node in a cell will be active and the counter is maintained for the available capacity of each outward link when the active node has data to send (whether from its own generated data or the received data). It will choose one of the available links to send the data to, after sending the capacity of the link is reduced by the amount of the data sent. When one node runs out of energy, the other node in cell will become active. When all the nodes in a cell die out, the cell is considered at a loss of coverage. The result of the linear programming problem can also be used to estimate the lifetime of the sensor network for choosing the proper network density.

\section{Evaluation Model}

In order to evaluate our proposed scheme, we have done simulation and compared with PEGASIS [7], under the same condition in the aspect of network lifetime. The linear problem is then solved using the public domain package LP_SOLVE. In the simulation environment, we formulate the sensor field of $30 * 30 \mathrm{~m}, 50 * 50 \mathrm{~m}$ with the sensing range of $R_{s}=10 \mathrm{~m}$, the number of sensors in the network is $50,70,100$ and the BS position is either at the corner of the field $(0,0)$ or at remote position $(15,70)$. The calculation for communication and computation energy consumption is based on the model discussed in [8]. The power consumption for transmitting is $E_{T x}(i, j)=c_{i j} f_{i j}$ and the power consumption for receiving $E_{R x}(i, j)=E_{l e c} f_{i j}$, where $c_{i j}=E_{l e c}+E_{a m p}\left(d_{i j}\right)^{2}$, $E_{l e c}=50 \mathrm{~nJ} /$ bit and $E_{a m p}=100 \mathrm{pJ} / \mathrm{bit} / \mathrm{m}^{2}$.

The PEGASIS that is compared with our proposed algorithm is an efficient chainbased routing protocol. In PEGASIS, the chain is formed among all the nodes in the network using greedy algorithm, the data is gathered and aggregated along the chain and then, forwarded to the BS. Here, we apply PEGASIS with the chain which is constructed among the active nodes of each cell in order to adopt the same condition of the proposed algorithm. When an active node runs out of energy, the other node in the cell is selected as the active node and is used in the chain.

The following table compares the result from the proposed scheme with PEGASIS in terms of coverage lifetime.

The result shows that the lifetime of the network under the solution obtained from the network flow of the LP is higher than that of the PEGASIS, in the case of low density as well as high density, and both when the BS is at the corner of the network or at the remote position for various network sizes. The higher the density of the network, the higher is the lifetime (which is nearly proportional to the increase in density). 
Table 2. Comparison of coverage lifetime between Proposed scheme and PEGASIS

\begin{tabular}{|c|c|c|c|c|}
\hline BS position & Area & No. of nodes & $\begin{array}{c}\text { 1st loss of } \\
\text { cell coverage } \\
\text { using LP }\end{array}$ & $\begin{array}{c}\text { 1st loss of } \\
\text { cell coverage } \\
\text { using } \\
\text { PEGASIS }\end{array}$ \\
\hline$(0,0)$ & $(30,30)$ & 15 & 542 & 443 \\
\hline$(15,70)$ & $(30,30)$ & 15 & 413 & 368 \\
\hline$(0,0)$ & $(30,30)$ & 70 & 1704 & 1161 \\
\hline$(15,70)$ & $(30,30)$ & 70 & 1477 & 1119 \\
\hline$(25,75)$ & $(50,50)$ & 50 & 617 & 455 \\
\hline$(25,75)$ & $(50,50)$ & 100 & 1137 & 753 \\
\hline
\end{tabular}

The result can be concluded that the solution of LP provides the optimal network flow as compared to the near optimal in the case of PEGASIS. Also as the number of sensor nodes increases the number of nodes in the cell increases and the distance among the nodes reduces, so that the lifetime of both PEGASIS and LP solution increases.

\section{Conclusion}

In this paper, we have applied the linear programming in obtaining the optimal transmission flow to maximize the lifetime of coverage for the sensor network. The solution of the problem provides the optimal network flow for the sensor networks under consideration. By dividing the network into grids, with one active node at a time in each cell to cover the whole cell, we can reduce the network flow problem to a manageable size and thus, can scale with the network with a large number of sensor nodes. The simulation result shows that the solution obtained provides better result than the near optimal routing protocol PEGASIS, in both low and high density network, and also in case, BS is at the corner or far away from the sensor field under different area size.

\section{References}

1. S. Slijepcevic and M. Potkonjak, "Power Efficient Organization of Wireless Sensor Networks”, Proc. of IEEE International Conference on Communications, Vol 2, pp 472476, Helsinki, Finland, June 2001.

2. M. Cardei, D. MacCallum, X. Cheng, M. Min, X. Jia, D. Li, and D.Z. Du, ”Wireless Sensor Networks with Energy Efficient Organization”, Journal of Interconnection Networks, Vol 3, No 34, pp 213229, Dec 2002.

3. J. Carle and D. Simplot, "Energy Efficient Area Monitoring by Sensor Networks", accepted to appear in IEEE Computer.

4. K. Chakrabarty, S. S. Iyengar, H. Qi and E. Cho, "Grid coverage for surveillance and target location in distributed sensor networks", IEEE Transactions on Computers, vol. 51, pp. 1448-1453, December 2002. 
5. S. Megerian and M. Potkonjak, "Low Power 0/1 Coverage and Scheduling Techniques in Sensor Networks”, UCLA Technical Reports 030001. January 2003.

6. F. Ye, G. Zhong, S. Lu, and L. Zhang, ”Energy Efficient Robust Sensing Coverage in Large Sensor Networks", Technical Report UCLA, 2002.

7. S. Lindsey, C. Raghavendra, and K. Sivalingam, "Data Gathering Algorithms in Sensor Networks Using the Energy Metrics", IEEE Transactions on Parallel and Distributed Systems, vol. 13, no. 9, Sep. 2002, pp. 924-935

8. Wendi Heinzelman, Anantha Chandrakasan, and Hari Balakrishnan, "Energy-Efficient Communication Protocols for Wireless Microsensor Networks", Proc. Hawaaian Int'l Conf. on Systems Science, January 2000.

9. S. Meguerdichian, F. Koushanfar, M. Potkonjak, M.B. Srivastava, "Coverage Problems in Wireless Ad-Hoc Sensor Networks", IEEE Infocom 2001,Vol 3, pp. 1380-1387, April 2001.

10. Konstantinos Kalpakis, Koustuv Dasgupta, and Parag Namjoshi, "Maximum Lifetime Data Gathering and Aggregation in Wireless Sensor Networks", In the Proceedings of the 2002 IEEE International Conference on Networking (ICN'02), Atlanta, Georgia, August 26-29, 2002.

11. J.H. Chang and L. Tassiulas, "Energy Conserving Routing in Wireless Ad-hoc Networks", Proc. IEEE INFOCOM 2000, Tel Aviv, Isreal, Mar. 2000.

12. M. Cardei and J. Wu, "Coverage in Wireless Sensor Networks", accepted to appear in Handbook of Sensor Networks, M.Ilyas (ed.), CRC Press, 2004

13. Dimitris Bertsimas and John N. Tsitsiklis, "Introduction to Linear Optimization”, Athena Scientific, Belmont, MA, 1997.

14. Operation Research: "Application and algorithm, Wayne L. Winston January”, 1994

15. D. Tian and N. D. Georganas, "A Coverage-Preserving Node Scheduling Scheme for Large Wireless Sensor Networks”, Proc. of the 1st ACM Workshop on Wireless Sensor Networks and Applications, 2002.

16. Y. Thomas Hou, Yi Shi, Jianping Pan, Alon Efrat, and Scott Midkiff, ”Optimal SingleSession Flow Routing for Wireless Sensor Networks", IEEE Vehicular Technology Conference, 2004. 